\title{
Clinical Significance of Skeletal Muscle Loss Following Lung Resection for Cancer: Recovery and Sarcopenia are Linked to Cancer Outcomes
}

\author{
Philip W. Carrott Jr, MD, FACS \\ Section of Thoracic Surgery, 2120N Taubman Center/5344, University of Michigan Health System, Ann Arbor, MI
}

Early-stage lung cancer has a low but real rate of recurrence and metastatic disease in the order of 10-20\%. Dr. Takamori and colleagues from Kyushu University have identified skeletal muscle loss following resection as a predictor of cancer-specific outcomes in lung cancer following resection. ${ }^{1}$ The relation of muscle mass and other physiologic markers seen on computed tomography (CT) scans has recently become a significant predictor of outcomes in a number of disease states and surgical populations. The field has been developed as 'morphomics' to identify these morphological characteristics of patients. ${ }^{2}$ Thus far, transplant patient outcomes and surgical outcomes have been reliably linked to patient morphomic factors such as psoas muscle area, and density of visceral and truncal fat, or dorsal muscle density in abdominal and thoracic CT scans. ${ }^{2}$ Cancer-specific outcomes have been shown in prior studies in adrenocortical cancers. ${ }^{3}$

The link between patient physiology and outcomes makes sense, but now linking the likelihood of cancerspecific outcomes to a patient's physiologic or morphomic status makes a further leap in the value of assessing these very specific factors, particularly in early-stage lung cancer. The authors nicely show a relationship between muscle loss and poorer respiratory status with disease-free and overall survival. These important factors should be followed and studied in future patients and disease states to better identify those patients who may benefit from close

(C) Society of Surgical Oncology 2018

First Received: 19 December 2017;

Published Online: 29 January 2018

P. W. Carrott Jr, MD, FACS

e-mail: pcarrott@med.umich.edu follow-up or perhaps postoperative nutritional intervention or physiotherapy.

The value in any new finding that can influence clinical course is how it can be applied to clinical care. These patients will typically have routine surveillance CT scans for recurrent or metastatic cancer. The morphomic data can also be relatively easily surveilled, and the subset of patients at risk might benefit from closer follow-up or enrollment in physical therapy or nutritional rehabilitation programs. These data may suggest to the treating physician which patients may benefit from adjuvant therapy, although these patients with significant sarcopenia would not be good candidates for high-morbidity treatments. Alternatively, those with significant sarcopenia on preoperative or pretreatment CT scans may have their treatment plans altered by either avoiding surgery altogether or avoiding minimal resection strategies such as wedge resection or segementectomy.

This also makes sense on a physiologic level, but one that I think is poorly understood and also difficult to discern, i.e. what makes a patient sarcopenic or predisposed to poor physiologic health and recurrent cancer? Is it nutrition? Patient conditioning, or immune status? In all likelihood, it is a combination of factors, as well as genetic and cancer-specific factors. Morphomics and CT scan variables are likely some of the most objective and specific metrics in medicine, and will do much to quantify a patient's real health status. Continuing to work to identify these relevant health factors and acting on them may produce gains in identifying health risks, when outward signs of disease are more subtle. I congratulate the authors on nicely outlining this link, even in early-stage disease.

\section{REFERENCES}

1. Takamori, S, Toyokawa, G, Okamoto, T, Shimokawa, M, Kinoshita, F, Kozuma, Y, et al. Clinical impact and risk factors 
for skeletal muscle loss after complete resection of early non-small cell lung cancer. Ann Surg Oncol. 2018. https://doi.org/10.1245/ s10434-017-6328-y.

2. Englesbe MJ, Lee JS, He K, Fan L, Schaubel DE, Sheetz KH, et al. Analytic morphomics, core muscle size, and surgical outcomes. Ann Surg. 2012; 256(2):255-61.
3. Miller BS, Ignatoski KM, Daignault S, Lindland C, Doherty M, Gauger PG, et al. Worsening central sarcopenia and increasing intra-abdominal fat correlate with decreased survival in patients with adrenocortical carcinoma. World J Surg. 2012;36(7):1509-16. 